\title{
NAIVE REALISM ABOUT OPERATORS
}

\author{
Martin Daumer, Detlef Dürr \\ Mathematisches Institut der Universität München \\ Theresienstraße 39, 80333 München, Germany \\ Sheldon Goldstein \\ Department of Mathematics, Rutgers University \\ New Brunswick, NJ 08903, USA \\ Nino Zanghì \\ Istituto di Fisica dell'Università di Genova, INFN \\ via Dodecaneso 33, 16146 Genova, Italy
}

January 11, 1996

\begin{abstract}
A source of much difficulty and confusion in the interpretation of quantum mechanics is a "naive realism about operators." By this we refer to various ways of taking too seriously the notion of operator-as-observable, and in particular to the all too casual talk about "measuring operators" that occurs when the subject is quantum mechanics. Without a specification of what should be meant by "measuring" a quantum observable, such an expression can have no clear meaning. A definite specification is provided by Bohmian mechanics, a theory that emerges from Schr̈odinger's equation for a system of particles when we merely insist that "particles" means particles. Bohmian mechanics clarifies the status and the role of operators as observables in quantum mechanics by providing the operational details absent from standard quantum mechanics. It thereby allows us to readily dismiss all the radical claims traditionally enveloping the transition from the classical to the quantum realm - for example, that we must abandon classical logic or classical probability. The moral is rather simple: Beware naive realism, especially about operators!
\end{abstract}

\section{Introduction}

Traditional naive realism is the view that the world is pretty much the way it seems, populated by objects which force themselves upon our attention as, and which in fact 
are, the locus of sensual qualities. A naive realist regards these "secondary qualities," for example color, as objective, as out there in the world, much as perceived. A decisive difficulty with this view is that once we understand, say, how our perception of what we call color arises, in terms of the interaction of light with matter, and the processing of the light by the eye, and so on, ${ }^{1}$ we realize that the presence out there of color per se would play no role whatsoever in these processes, that is, in our understanding what is relevant to our perception of "color." At the same time, we may also come to realize that there is, in the description of an object provided by the scientific world-view, as represented say by classical physics, nothing which is genuinely "color-like."

We shall argue that the basic problem with quantum theory, more fundamental than the measurement problem and all the rest, is a naive realism about operators, a fallacy which we believe is far more serious than traditional naive realism: With the latter we are deluded partly by language but in the main by our senses, in a manner which can scarcely be avoided without a good deal of scientific or philosophical sophistication; with the former we are seduced by language alone, to accept a view which can scarcely be taken seriously without a large measure of (what often passes for) sophistication.

The classical physical observables - for a system of particles, their positions $q=\left(\mathbf{q}_{k}\right)$, their momenta $p=\left(\mathbf{p}_{k}\right)$, and the functions thereof, i.e., functions on phase space -form a commutative algebra. It is generally taken to be the essence of quantization, the procedure which converts a classical theory to a quantum one, that $q, p$, and hence all functions $f(q, p)$ thereof are replaced by appropriate operators, on a Hilbert space, of possible wave functions, associated with the system under consideration. Thus quantization leads to a noncommutative operator algebra of "observables." Moreover, the fact that the observables in quantum theory form a noncommutative structure has traditionally been regarded as endowed with deep epistemological and or metaphysical significance and has variously been interpreted as the mathematical embodiment of irreducible indeterminacy or uncertainty and intrinsic fuzziness.

By naive realism about operators we refer to various, not entirely sharply defined, ways of taking too seriously the notion of operator-as-observable, and in particular to the all too casual talk about "measuring operators" which tends to occur as soon as a physicist enters quantum mode. What, after all, is meant by measuring an operator? If this is to have a meaning, that meaning must be supplied - it is not at all expressed by these words as they are normally understood. But more on this later.

Not many physicists - or for that matter philosophers - have focused on the issue of naive realism about operators, but Schrödinger and Bell have expressed similar or related concerns:

... the new theory [quantum theory] ... considers the [classical] model suitable for guiding us as to just which measurements can in principle be made on the relevant natural object. ... Would it not be pre-established harmony of a peculiar sort if the classical-epoch researchers, those who, as we hear today, had no idea of what measuring truly is, had unwittingly gone on to give us 
as legacy a guidance scheme revealing just what is fundamentally measurable for instance about a hydrogen atom!? (Schrödinger 1935)

Here are some words which, however legitimate and necessary in application, have no place in a formulation with any pretension to physical precision: system; apparatus; environment; microscopic, macroscopic; reversible, irreversible; observable; information; measurement.

... The notions of "microscopic" and "macroscopic" defy precise definition. ... Einstein said that it is theory which decides what is "observable". I think he was right. ... "observation" is a complicated and theory-laden business. Then that notion should not appear in the formulation of fundamental theory. ...

On this list of bad words from good books, the worst of all is "measurement". It must have a section to itself. (Bell 1990)

We agree almost entirely with Bell here. We insist, however, that "observable" is just as bad as "measurement," maybe even a little worse. Be that as it may, after listing Dirac's measurement postulates Bell continues:

It would seem that the theory is exclusively concerned about "results of measurement", and has nothing to say about anything else. What exactly qualifies some physical systems to play the role of "measurer"? Was the wavefunction of the world waiting to jump for thousands of millions of years until a single-celled living creature appeared? Or did it have to wait a little longer, for some better qualified system ... with a Ph.D.? If the theory is to apply to anything but highly idealized laboratory operations, are we not obliged to admit that more or less "measurement-like" processes are going on more or less all the time, more or less everywhere. Do we not have jumping then all the time?

The first charge against "measurement", in the fundamental axioms of quantum mechanics, is that it anchors the shifty split of the world into "system" and "apparatus". A second charge is that the word comes loaded with meaning from everyday life, meaning which is entirely inappropriate in the quantum context. When it is said that something is "measured" it is difficult not to think of the result as referring to some preexisting property of the object in question. This is to disregard Bohr's insistence that in quantum phenomena the apparatus as well as the system is essentially involved. If it were not so, how could we understand, for example, that "measurement" of a component of "angular momentum" ... in an arbitrarily chosen direction ... yields one of a discrete set of values? When one forgets the role of the apparatus, as the word "measurement" makes all too likely, one despairs of ordinary logic ... hence "quantum logic". When one remembers the role of the apparatus, ordinary logic is just fine. 
In other contexts, physicists have been able to take words from ordinary language and use them as technical terms with no great harm done. Take for example the "strangeness", "charm", and "beauty" of elementary particle physics. No one is taken in by this "baby talk".... Would that it were so with "measurement". But in fact the word has had such a damaging effect on the discussion, that I think it should now be banned altogether in quantum mechanics. (Ibid.)

While Bell focuses directly here on the misuse of the word "measurement" rather than on that of "observable," it is worth noting that the abuse of "measurement" is in a sense inseparable from that of "observable," i.e., from naive realism about operators. After all one would not be very likely to speak of measurement unless one thought that something, some "observable" that is, was somehow there to be measured.

More Bell:

The concept of 'measurement' becomes so fuzzy on reflection that it is quite surprising to have it appearing in physical theory at the most fundamental level. Less surprising perhaps is that mathematicians, who need only simple axioms about otherwise undefined objects, have been able to write extensive works on quantum measurement theory - which experimental physicists do not find it necessary to read.... Does not any analysis of measurement require concepts more fundamental than measurement? And should not the fundamental theory be about these more fundamental concepts? (Bell 1981)

... in physics the only observations we must consider are position observations, if only the positions of instrument pointers. It is a great merit of the de Broglie-Bohm picture to force us to consider this fact. If you make axioms, rather than definitions and theorems, about the 'measurement' of anything else, then you commit redundancy and risk inconsistency. (Bell 1982)

If our feeling that Bell's words are thoroughly compelling were widely shared, by physicists and philosophers, there would perhaps be little point in continuing this paper. But it is not, so we continue! Moreover, we wish in any case to focus on what Bell calls "the de Broglie-Bohm picture" - what we prefer to call Bohmian mechanics - for the light it sheds on naive realism about operators.

We wish to do two things here: We wish to elaborate on why we think what we have called naive realism about operators (taking operators too seriously as observables) is bad, and we wish to relate this issue to Bohmian mechanics. Briefly stated, the relevant connections between naive realism about operators and Bohmian mechanics are the following:

1. A frequent complaint about Bohmian mechanics, in which positions play a fundamental role, is expressed in terms of questions like "What about other observables?" 
2. Bohmian mechanics allows the inadequacy, indeed the utter wrongheadedness, of naive realism about operators to emerge with stark clarity.

\section{Bohmian mechanics}

According to orthodox quantum theory, the complete description of a system of particles is provided by its wave function. It is rarely noticed that even this statement is somewhat problematical: If "particles" is intended with its usual meaning-point-like entities whose most important feature is their positions in space - the statement is clearly false, since the complete description would then have to include these positions; otherwise, the statement is, to be charitable, vague. Bohmian mechanics is the theory which emerges when we indeed insist that "particles" means particles.

According to Bohmian mechanics (Bohm 1952, Bohm et al. 1993, Bell 1987, Dürr et al. 1992, 1996, Holland 1993, Berndl et al. 1995), the complete description of an $N$-particle system is provided by its wave function $\psi$ and its configuration $Q=\left(\mathbf{Q}_{1}, \ldots, \mathbf{Q}_{N}\right)$, where the $\mathbf{Q}_{k}$ are the positions of the particles. The wave function, which evolves according to Schrödinger's equation, choreographs the motion of the particles: these evolve - in the simplest manner possible - according to a first-order ordinary differential equation

$$
\frac{d Q}{d t}=v^{\psi}(Q)
$$

whose right-hand side, a velocity vector field on configuration space, is generated by the wave function. Considerations of space-time symmetry - Galilean and time-reversal invariance then determine the form of $v^{\psi}=\left(\mathbf{v}_{1}^{\psi}, \ldots, \mathbf{v}_{N}^{\psi}\right)$ (Dürr et al. 1992), and we arrive at the defining (evolution) equations of Bohmian mechanics:

$$
\frac{d \mathbf{Q}_{k}}{d t}=\mathbf{v}_{k}^{\psi}\left(\mathbf{Q}_{1}, \ldots, \mathbf{Q}_{N}\right) \equiv \frac{\hbar}{m_{k}} \operatorname{Im} \frac{\boldsymbol{\nabla}_{\mathbf{q}_{k}} \psi}{\psi}\left(\mathbf{Q}_{1}, \ldots, \mathbf{Q}_{N}\right)
$$

and

$$
i \hbar \frac{\partial \psi}{\partial t}=H \psi
$$

where $H$ is the usual Schrödinger Hamiltonian, containing as parameters the masses $m_{1}, \ldots, m_{N}$ of the particles as well as the potential energy function $V$ of the system.

For an $N$-particle universe, these two equations form a complete specification of the theory. There is no need, and indeed no room, for any further axioms, describing either the behavior of "other observables" or the effects of "measurement."

Bohmian mechanics is the most naively obvious embedding imaginable of Schrödinger's equation into a completely coherent physical theory! If one didn't already know better, 
one would naturally conclude that it can't "work," i.e., that it can't account for quantum phenomena. After all, if something so obvious and, indeed, so trivial works, great physicists would never have insisted, as they have and as they continue to do, that quantum theory demands radical epistemological and metaphysical innovations.

Moreover, when we think about it, how could Bohmian mechanics have much to do with quantum theory? Where is quantum randomness in this deterministic theory? Where is quantum uncertainty? Where are operators as observables and all the rest?

Be that as it may, Bohmian mechanics is certainly $a$ theory. It describes a world in which particles move in a highly non-Newtonian sort of way, and it would do so even were it the case that the way they do move in this theory had absolutely nothing to do with quantum mechanics.

It turns out, however, that a remarkable consequence (!) of the equations (2.1) and (2.2) is that when a system has wave function $\psi$ its configuration is random, with probability density $\rho$ given typically by $\rho=|\psi|^{2}$, the quantum equilibrium distribution. In other words, it turns out that systems are somehow typically in quantum equilibrium. Moreover, this conclusion comes together with the clarification of what precisely this means, and also implies that a Bohmian universe embodies an absolute uncertainty which can itself be regarded as the origin of the uncertainty principle. We shall not go into these matters here, having discussed them at length elsewhere (Dürr et al. 1992, 1996). We note, however, that nowadays, with chaos theory and nonlinear dynamics so fashionable, it is not generally regarded as terribly astonishing for an appearance of randomness to emerge from a deterministic dynamical system.

It also turns out that the entire quantum formalism, operators as observables and all the rest, is a consequence of Bohmian mechanics, and since this is relevant to the issue of naive realism about operators, we do wish to spend some time sketching how this comes about.

\section{The quantum formalism}

Information about a system does not spontaneously pop into our heads, or into our (other) "measuring" instruments; rather, it is generated by an experiment: some physical interaction between the system of interest and these instruments, which together (if there is more than one) comprise the apparatus for the experiment. Moreover, this interaction is defined by, and must be analyzed in terms of, the physical theory governing the behavior of the composite formed by system and apparatus. If the apparatus is well designed, the experiment should somehow convey significant information about the system. However, we cannot hope to understand the significance of this "information"-for example, the nature of what it is, if anything, that has been measured - without some such theoretical analysis.

Whatever its significance, the information conveyed by the experiment is registered in the apparatus as an output, represented, say, by the orientation of a pointer. Moreover, 
when we speak of an experiment, we have in mind a fairly definite initial state of the apparatus, the ready state, one for which the apparatus should function as intended, and in particular one in which the pointer has some "null" orientation.

For Bohmian mechanics we should expect in general that, as a consequence of the quantum equilibrium hypothesis, the outcome of the experiment - the final pointer orientation - will be random: Even if the system-apparatus composite initially has a definite, known wave function, so that the outcome is determined by the initial configuration of system and apparatus, this configuration is random, since the composite system is in quantum equilibrium, i.e., the distribution of this configuration is given by $|\Psi(x, y)|^{2}$, where $\Psi$ is the wave function of the system-apparatus composite and $x$, respectively $y$, is the generic system, respectively apparatus, configuration. There are, however, special experiments whose outcomes are somewhat less random than we might have thought possible.

In fact, consider a measurement-like experiment, one which is reproducible in the sense that it will yield the same outcome as originally obtained if it is immediately repeated. (Note that this means that the apparatus must be immediately reset to its ready state, or a fresh apparatus must be employed, while the system is not tampered with so that its initial state for the repeated experiment is its final state produced by the first experiment.) Suppose that this experiment admits, i.e., that the apparatus is so designed that there are, only a finite (or countable) number of possible outcomes $\alpha{ }^{2}$ for example, $\alpha=$ "left" and $\alpha=$ "right". The experiment also usually comes equipped with a calibration $\lambda_{\alpha}$, an assignment of numerical values (or a vector of such values) to the various outcomes $\alpha$.

It can be shown (Daumer et al. 1996), under further simplifying assumptions, that for such reproducible experiments there are special subspaces $\mathcal{H}_{\alpha}$ of the system Hilbert space $\mathcal{H}$ of (initial) wave functions, which are mutually orthogonal and span the entire system Hilbert space

$$
\mathcal{H}=\bigoplus_{\alpha} \mathcal{H}_{\alpha}
$$

such that if the system's wave function is initially in $\mathcal{H}_{\alpha}$, outcome $\alpha$ definitely occurs and the value $\lambda_{\alpha}$ is thus definitely obtained. It then follows that for a general initial system wave function

$$
\psi=\sum_{\alpha} \psi_{\alpha} \equiv \sum_{\alpha} P_{\mathcal{H}_{\alpha}} \psi
$$

where $P_{\mathcal{H}_{\alpha}}$ is the projection onto the subspace $\mathcal{H}_{\alpha}$, the outcome $\alpha$ is obtained with (the usual) probability ${ }^{3}$

$$
p_{\alpha}=\left\|P_{\mathcal{H}_{\alpha}} \psi\right\|^{2} .
$$

In particular, the expected value obtained is

$$
\sum_{\alpha} p_{\alpha} \lambda_{\alpha}=\sum_{\alpha} \lambda_{\alpha}\left\|P_{\mathcal{H}_{\alpha}} \psi\right\|^{2}=\langle\psi, A \psi\rangle
$$


where

$$
A=\sum_{\alpha} \lambda_{\alpha} P_{\mathcal{H}_{\alpha}}
$$

and $\langle\cdot, \cdot\rangle$ is the usual inner product:

$$
\langle\psi, \phi\rangle=\int \psi^{*}(x) \phi(x) d x .
$$

What we wish to emphasize here is that, insofar as the statistics for the values which result from the experiment are concerned, the relevant data for the experiment are the collection $\left(\mathcal{H}_{\alpha}\right)$ of special subspaces, together with the corresponding calibration $\left(\lambda_{\alpha}\right)$, and this data is compactly expressed and represented by the self-adjoint operator $A$, on the system Hilbert space $\mathcal{H}$, given by (3.5). Thus with a reproducible experiment $\mathfrak{E}$ we naturally associate an operator $A=A_{\mathfrak{E}}$,

$$
\mathfrak{E} \mapsto A,
$$

a single mathematical object, defined on the system alone, in terms of which an efficient description of the possible results is achieved. If we wish we may speak of operators as observables, but if we do so it is important that we appreciate that in so speaking we merely refer to what we have just sketched: the role of operators in the description of certain experiments. ${ }^{4}$

In particular, so understood, the notion of operator-as-observable in no way implies that anything is measured in the experiment, and certainly not the operator itself! In a general experiment no system property is being measured, even if the experiment happens to be measurement-like. (Position measurements are of course an important exception.) What in general is going on in obtaining outcome $\alpha$ is completely straightforward and in no way suggests, or assigns any substantive meaning to, statements to the effect that, prior to the experiment, observable $A$ somehow had a value $\lambda_{\alpha}$ - whether this be in some determinate sense or in the sense of Heisenberg's "potentiality" or some other ill-defined fuzzy sense - which is revealed, or crystallized, by the experiment. 5

Much of the preceding sketch of the emergence and role of operators as observables in Bohmian mechanics, including of course the von Neumann-type picture of "measurement" at which we arrive, applies as well to orthodox quantum theory. In fact, it would appear that the argument against naive realism about operators provided by such an analysis has even greater force from an orthodox perspective: Given the initial wave function, at least in Bohmian mechanics the outcome of the particular experiment is determined by the initial configuration of system and apparatus, while for orthodox quantum theory there is nothing in the initial state which completely determines the outcome. Indeed, we find it rather surprising that most proponents of the von Neumann analysis of measurement, beginning with von Neumann, nonetheless seem to retain their naive realism about operators. Of course, this is presumably because more urgent matters - the measurement 
problem and the suggestion of inconsistency and incoherence that it entails - soon force themselves upon one's attention. Moreover such difficulties perhaps make it difficult to maintain much confidence about just what should be concluded from the "measurement" analysis, while in Bohmian mechanics, for which no such difficulties arise, what should be concluded is rather obvious. 6

\section{The reality of spin and other observables}

The canonical example of a "quantum measurement" is provided by the Stern-Gerlach experiment. We wish to focus on this example here in order to make our previous considerations more concrete, as well as to present some further considerations about the "reality" of operators-as-observables. We wish in particular to comment on the status of spin. We shall therefore consider a Stern-Gerlach "measurement" of the spin of an electron, even though such an experiment is generally believed to be unphysical (Mott 1929), rather than of the internal angular momentum of a neutral atom.

We must first explain how to incorporate spin into Bohmian mechanics. This is very easy; we need do, in fact, almost nothing: Our derivation of Bohmian mechanics (Dürr et al. 1992) was based in part on rotation invariance, which requires in particular that rotations act on the value space of the wave function. The latter is rather inconspicuous for spinless particles - with complex-valued wave functions, what we have been considering up till now - since rotations then act in a trivial manner on the value space $\mathbb{C}$. The simplest nontrivial (projective) representation of the rotation group is the 2-dimensional, "spin $\frac{1}{2}$ " representation; this representation leads to a Bohmian mechanics involving spinor-valued wave functions for a single particle and spinor-tensor-product-valued wave function for many particles. Thus the wave function of a single spin- $-\frac{1}{2}$ particle has two components

$$
\psi(\mathbf{q})=\left(\begin{array}{l}
\psi_{1}(\mathbf{q}) \\
\psi_{2}(\mathbf{q})
\end{array}\right)
$$

which get mixed under rotations according to the action generated by the Pauli spin matrices $\boldsymbol{\sigma}=\left(\sigma_{x}, \sigma_{y}, \sigma_{z}\right)$, which may be taken to be

$$
\sigma_{x}=\left(\begin{array}{cc}
0 & 1 \\
1 & 0
\end{array}\right) \quad \sigma_{y}=\left(\begin{array}{cc}
0 & -i \\
i & 0
\end{array}\right) \quad \sigma_{z}=\left(\begin{array}{cc}
1 & 0 \\
0 & -1
\end{array}\right)
$$

Beyond the fact that the wave function now has a more abstract value space, nothing changes from our previous description: The wave function evolves via (2.2), where now the Hamiltonian $H$ contains the Pauli term, for a single particle proportional to $\mathbf{B} \cdot \boldsymbol{\sigma}$, which represents the coupling between the "spin" and an external magnetic field B. The configuration evolves according to the natural extension of (2.1) to spinors, obtained say by multiplying both the numerator and denominator of the argument of "Im" on the left by $\psi^{*}$ and interpreting the result for the case of spinor values as a spinor-inner-product. 
Let's focus now on a Stern-Gerlach "measurement of $A=\sigma_{z}$." An inhomogeneous magnetic field is established in a neighborhood of the origin, by means of a suitable arrangement of magnets. This magnetic field is oriented more or less in the positive $z$ direction, and is increasing in this direction. We also assume that the arrangement is invariant under translations in the $x$-direction, i.e., that the geometry does not depend upon $x$-coordinate. An electron, with a fairly definite momentum, is directed towards the origin along the negative $y$-axis. Its passage through the inhomogeneous field generates a vertical deflection of its wave function away from the $y$-axis, which for Bohmian mechanics leads to a similar deflection of the electron's trajectory. If its wave function $\psi$ were initially an eigenstate of $\sigma_{z}$ of eigenvalue $1(-1)$, i.e., if it were of the form

$$
\psi=|\uparrow\rangle \otimes \phi_{0} \quad\left(\psi=|\downarrow\rangle \otimes \phi_{0}\right)
$$

where

$$
|\uparrow\rangle=\left(\begin{array}{l}
1 \\
0
\end{array}\right) \quad \text { and } \quad|\downarrow\rangle=\left(\begin{array}{l}
0 \\
1
\end{array}\right) \text {, }
$$

then the deflection would be in the positive (negative) $z$-direction (by a rather definite angle). For a more general initial wave function, passage through the magnetic field will, by linearity, split the wave function into an upward-deflected piece (proportional to $|\uparrow\rangle$ ) and a downward-deflected piece (proportional to $|\downarrow\rangle$ ), with corresponding deflections of the possible trajectories.

The outcome is registered by detectors placed in the way of these two "beams." Thus of the four kinematically possible outcomes ("pointer positions") the occurrence of no detection defines the null output, simultaneous detection is irrelevant ( since it does not occur if the experiment is performed one particle at a time), and the two relevant outcomes correspond to registration by either the upper or the lower detector. Thus the calibration for a measurement of $\sigma_{z}$ is $\lambda_{\text {up }}=1$ and $\lambda_{\text {down }}=-1$ (while for a measurement of the $z$-component of the spin angular momentum itself the calibration is the product of what we have just described by $\left.\frac{1}{2} \hbar\right)$.

Note that one can completely understand what's going on in this Stern-Gerlach experiment without invoking any additional property of the electron, e.g., its actual z-component of spin that is revealed in the experiment. For a general initial wave function there is no such property; what is more, the transparency of the analysis of this experiment makes it clear that there is nothing the least bit remarkable (or for that matter "nonclassical") about the nonexistence of this property. As we emphasized earlier, it is naive realism about operators, and the consequent failure to pay attention to the role of operators as observables, i.e., to precisely what we should mean when we speak of measuring operatorobservables, that creates an impression of quantum peculiarity.

Bell has said that (for Bohmian mechanics) spin is not real. Perhaps he should better have said: "Even spin is not real," not merely because of all observables, it is spin which is 
generally regarded as quantum mechanically most paradigmatic, but also because spin is treated in orthodox quantum theory very much like position, as a "degree of freedom" a discrete index that supplements the continuous degrees of freedom corresponding to position - in the wave function. Be that as it may, his basic meaning is, we believe, this: Unlike position, spin is not primitive, ${ }^{7}$ i.e., no actual discrete degrees of freedom, analogous to the actual positions of the particles, are added to the state description in order to deal with "particles with spin." Roughly speaking, spin is merely in the wave function. At the same time, as just said, "spin measurements" are completely clear, and merely reflect the way spinor wave functions are incorporated into a description of the motion of configurations.

It might be objected that while spin may not be primitive, so that the result of our "spin measurement" will not reflect any initial primitive property of the system, nonetheless this result is determined by the initial configuration of the system, i.e., by the position of our electron, together with its initial wave function, and as such - as a function $X_{\sigma_{z}}(\mathbf{q}, \psi)$ of the state of the system-it is some property of the system and in particular it is surely real. Concerning this, several comments.

The function $X_{\sigma_{z}}(\mathbf{q}, \psi)$, or better the property it represents, is (except for rather special choices of $\psi$ ) an extremely complicated function of its arguments; it is not "natural," not a "natural kind": It is not something in which, in its own right, we should be at all interested, apart from its relationship to the result of this particular experiment.

Be that as it may, it is not even possible to identify this function $X_{\sigma_{z}}(\mathbf{q}, \psi)$ with the measured spin component, since different experimental setups for "measuring the spin component" may lead to entirely different functions. In other words $X_{\sigma_{z}}(\mathbf{q}, \psi)$ is an abuse of notation, since the function $X$ should be labeled, not by $\sigma_{z}$, but by the particular experiment for "measuring $\sigma_{z}$ ".

For example (Albert 1992, p.153), if $\psi$ and the magnetic field have sufficient reflection symmetry with respect to a plane between the poles of our SG magnet, and if the magnetic field is reversed, then the sign of what we have called $X_{\sigma_{z}}(\mathbf{q}, \psi)$ will be reversed: for both orientations of the magnetic field the electron cannot cross the plane of symmetry and hence if initially above respectively below the symmetry plane it remains above respectively below it. But when the field is reversed so must be the calibration, and what we have denoted by $X_{\sigma_{z}}(\mathbf{q}, \psi)$ changes sign with this change in experiment. ${ }^{8}$

In general $X_{A}$ does not exist, i.e., $X_{\mathfrak{E}}$, the result of the experiment $\mathfrak{E}$, in general depends upon $\mathfrak{E}$ and not just upon $A=A_{\mathfrak{E}}$, the operator associated with $\mathfrak{E}$. In foundations of quantum mechanics circles this situation is referred to as contextuality, but we believe that this terminology, while quite appropriate, somehow fails to convey with sufficient force the rather definitive character of what it entails: Properties that are merely contextual are not properties at all; they do not exist, and their failure to do so is in the strongest sense possible! We thus believe that contextuality reflects little more than the rather obvious observation that the result of an experiment should depend upon how it is performed!

We summarize our comparison of the status of position with that of other observables 
in the following chart:

\begin{tabular}{|c|c|}
\hline position & other observables \\
\hline \hline real & not real \\
\hline primitive & not primitive \\
\hline natural (kind) & not natural (kind) \\
\hline noncontextual & contextual \\
\hline
\end{tabular}

\section{$5 \quad$ Hidden variables}

What about the "no-go" theorems for hidden variables? ${ }^{9}$ These theorems show that there is no "good" map $A \mapsto X_{A}$ from operators to random variables (on the space of "hidden variables"), where by "good" we mean in the sense that the joint distributions of the random variables are consistent with the corresponding quantum mechanical distributions whenever the latter are defined.

As commonly understood, these theorems involve a certain irony: They conclude with the impossibility of a deterministic description, or more generally of any sort of realist description, only by in effect themselves assuming a "realism" of a most implausible variety, namely, naive realism about operators. For why else would a realist, even one who is also a determinist, expect there to be such a map? After all, the fact that the same operator plays a role in different experiments does not imply that these experiments have much else in common, and certainly not that they involve measurements of the same thing. It is thus with detailed experiments, and not with the associated operators, that random variables might reasonably be expected to be associated.

When faced with the inconsistency of possible values as expressed by the "no-go" theorems, how should one respond? As does a "typical" physicist, by declaring in effect that quantum mechanics does not allow us to ask the obvious questions? But even if we should chose to forbid ourselves from asking sufficiently many questions to notice it, the state of affairs described by the theorems nontheless logically implies the obvious conclusion, namely, that the incompatible joint values refer to different, and incompatible, experimental set-ups, just as Bohr told us all along. This mathematical incompatibility of "joint values" thus seems to attain genuine physical significance only to the extent that we are seduced by naive realism about operators. ${ }^{10}$

Referring to the axioms involved in the no-hidden-variables theorems, Bell says:

A final moral concerns terminology. Why did such serious people take so seriously axioms which now seem so arbitrary? (Bell 1982)

To this question we are tempted to respond that the answer, of course, is that these "serious people" were deluded by naive realism about operators. However, what Bell is 
really asking is why they should have been so deluded, as is made clear by what he says next:

I suspect that they were misled by the pernicious misuse of the word 'measurement' in contemporary theory. This word very strongly suggests the ascertaining of some preexisting property of some thing, any instrument involved playing a purely passive role. Quantum experiments are just not like that, as we learned especially from Bohr. The results have to be regarded as the joint product of 'system' and 'apparatus,' the complete experimental set-up. But the misuse of the word 'measurement' makes it easy to forget this and then to expect that the 'results of measurements' should obey some simple logic in which the apparatus is not mentioned. The resulting difficulties soon show that any such logic is not ordinary logic.

Note, in particular, the sentence that ends with "in which the apparatus is not mentioned." This makes little sense without an implicit reference to naive realism about operators: Everyone would agree that, even if it were not necessary to mention the apparatus per se, at least something would have to be mentioned; Bell is here criticizing the view that for this "something," the operator-as-observable that is being "measured" should suffice.

Bell continues:

It is my impression that the whole vast subject of 'Quantum Logic' has arisen in this way from the misuse of a word. I am convinced that the word 'measurement' has now been so abused that the field would be significantly advanced by banning its use altogether, in favour for example of the word 'experiment.'

\section{Comments}

Let's reconsider a fragment of one of our previous Bell quotations:

If you make axioms, rather than definitions and theorems, about the 'measurement' of anything else [other than position], then you commit redundancy and risk inconsistency.

We would like to propose what we believe to be a small improvement. Replace "measurement" by "behavior." Then add to "redundancy" and "inconsistency" the further possibility of irrelevance. In other words we are proposing the following amendment:

If you make axioms, rather than definitions and theorems, about the behavior of anything else -beyond what is required to fully specify the behavior of positions - then either you commit redundancy and risk inconsistency, or you commit irrelevancy

For example, suppose we add, say to Bohmian mechanics, some axioms governing the behavior of "momentum." Then there are two possibilities: 
1. On the one hand, by "momentum" we may mean, say, mass times velocity, in which case we have either redundancy or inconsistency.

2. On the other hand, if "momentum" is not given a meaning in terms of the behavior of configurations - if it is a brand new property as it were - then it is irrelevant!

A related lesson of Bohmian mechanics is one of flexibility: Not only need we not consider "other observables" on a fundamental level, it is not even necessary that the primitive variables (what the theory is fundamentally about) - in Bohmian mechanics the positions of the particles - be "observables" in the sense that they are associated with self-adjoint operators in the usual way. That they are for Bohmian mechanics is best regarded as an accident arising from incidental features (for example, the form of the inner product) of the mathematical structure of nonrelativistic quantum theory. ${ }^{11}$

Let's now return to the objection, "What about other observables?" Since operators as observables are nothing more than a convenient mathematical device for describing what is most relevant about certain special experiments, asking this question amounts to nothing more than asking, "What about special experiments?" But put this way, there is no longer any suggestion of inadequacy or incompleteness.

\section{Other interpretations}

In this brief section we wish to outline how some of the more familiar interpretations of quantum theory fare with regard to the fallacy of naive realism about operators. We do so in the following chart:

\begin{tabular}{|c|c|}
\hline guilty & not guilty \\
\hline \hline Copenhagen (quantum orthodoxy) & Copenhagen (Bohr) \\
\hline many worlds $^{12}$ & many worlds $^{13}$ \\
\hline quantum logic $^{14}$ & many minds $^{15}$ \\
\hline quantum probability $^{16}$ & spontaneous localization $^{17}$ \\
\hline modal interpretation $^{18}$ & stochastic mechanics $^{19}$ \\
\hline consistent histories $^{20}$ & Bohmian mechanics \\
\hline
\end{tabular}

Note that, as is so often the case, the Copenhagen interpretation is hard to pin down! 


\section{Diatribe}

Why should we (continue to) insist upon a metaphysics - that observables or properties should somehow be identified with operators - which, while seeming to express the essential innovation of quantum theory, in fact conflicts (or at least is strongly at odds with) the very mathematical structure of the theory itself? What is the point of multiplying properties, new properties irreducible to what we have already, when their mutual incompatibility has been enshrined in quantum orthodoxy from its very inception (the uncertainty principle, complementarity); when the no-hidden-variables theorems establish their joint impossibility; so that in order to save them one must resort to such expedients, contortions, and perversions as quantum logic and quantum probability (or, at best, to something like van Fraassen's modal interpretation of quantum theory (van Fraassen 1991), with all the enormous complexity its formulation requires); when they add nothing of substance or value to our understanding of the use of operators as "observables" - of the role of operators in quantum theory - which is in fact quite straightforward, as a compact expression of the most important or relevant features of certain experiments, the analysis of which reveals that what is going on during such experiments is in general not a measurement of the associated operator - what would that mean anyway? - nor, indeed, of anything else worth mentioning!?

\section{Acknowledgments}

We are grateful to Rebecca Goldstein for her suggestions. This work was supported in part by NSF Grant No. DMS-9504556, by the DFG, and by the INFN.

\section{Notes}

1 That we don't understand the last link in the causal chain leading to our conscious perception is not very relevant here.

2 This is really no assumption at all, since the outcome should ultimately be converted to digital form, whatever its initial representation may be.

3 In the simplest such situation the unitary evolution for the wave function of the composite system carries the initial wave function $\Psi_{i}=\psi \otimes \Phi_{0}$ to the final wave function $\Psi_{f}=\sum_{\alpha} \psi_{\alpha} \otimes \Phi_{\alpha}$, where $\Phi_{0}$ is the ready apparatus wave function, and $\Phi_{\alpha}$ is the apparatus wave function corresponding to outcome $\alpha$. Then integrating $\left|\Psi_{f}\right|^{2}$ over $\operatorname{supp} \Phi_{\alpha}$, we immediately arrive at (3.3).

4 Operators as observables also naturally convey information about the system's wave function after the experiment. For example, for an ideal measurement, when the outcome is $\alpha$ the wave function of the system after the experiment is (proportional to) $P_{\mathcal{H}_{\alpha}} \psi$.

${ }^{5}$ Even speaking of the observable $A$ as having value $\lambda_{\alpha}$ when the system's wave function is in $\mathcal{H}_{\alpha}$, i.e., when this wave function is an eigenstate of $A$ of eigenvalue $\lambda_{\alpha}$, insofar as it suggests that something 
peculiarly quantum is going on when the wave function is not an eigenstate whereas in fact there is nothing the least bit peculiar about the situation, perhaps does more harm than good.

6 It might be objected that we are claiming to arrive at the quantum formalism under somewhat unrealistic assumptions, such as, for example, reproducibility. (We note in this regard that many more experiments than those satisfying our assumptions can be associated with operators in exactly the manner we have described.) We agree. But this objection misses the point of the exercise. The quantum formalism itself is an idealization; when applicable at all, it is only as an approximation. Beyond illuminating the role of operators as ingredients in this formalism, our point was to indicate how naturally it emerges. In this regard we must emphasize that the following question arises for quantum orthodoxy, but does not arise for Bohmian mechanics: For precisely which theory is the quantum formalism an idealization? (For further elaboration on this point, as well as for a discussion of how "generalized observables" (Davies, 1976) naturally arise in Bohmian mechanics, see Dürr et al. 1996 and Daumer et al. 1996.)

7 We should probably distinguish two senses of "primitive": i) the strongly primitive variables, which describe what the theory is fundamentally about, and ii) the weakly primitive variables, the basic variables of the theory, those which define the complete state description. The latter may either in fact be strongly primitive, or, like the electromagnetic field in classical electrodynamics, they may be required in order to express the laws which govern the behavior of the strongly primitive variables in a simple and natural way. While this probably does not go far enough - we should further distinguish those weakly primitive variables which, like the velocity, are functions of the trajectory of the strongly primitive variables, and those, again like the electromagnetic field, which are not-these details are not relevant to our present purposes, so we shall ignore these distinctions.

8 The change in experiment proposed by Albert is that "the hardness box is flipped over." However, with regard to spin this change will produce essentially no change in $X$ at all. To obtain the reversal of sign, either the polarity or the geometry of the SG magnet must be reversed, but not both.

9 The classical references on this topic are: von Neumann 1932, Gleason 1957, Jauch et al. 1963, Kochen et al. 1967. For a critical overview see Bell 1966, 1982.

10 This is perhaps a bit too strong: As is well known, Bell (Bell 1964) has shown that no-hidden-variablestype arguments, suitably applied, can be used to establish the rather striking physical conclusion that nature is nonlocal.

11 For some steps in the direction of the formulation of a Lorentz invariant Bohmian theory, as well as some reflections on the problem of Lorentz invariance, see Berndl et al. 1996.

12 Everett 1957. See also De Witt et al. (eds.) 1973.

13 We are referring here to Bell's reformulation of Everett's theory (Bell 1981).

14 'Quantum logic' was proposed by Birkhoff and von Neumann (Birkhoff et al. 1936). For more recent presentations and developments, see, e.g., Jauch 1968, and Beltrametti et al. 1981.

15 See Albert 1992.

16 That quantum mechanics has to do with a sort of 'noncommutative' probability originated probably with von Neumann 1932. A comprehensive list of the recent literature would probably be out of place here.

17 We are referring to the so called GRW-theory (Ghirardi et al. 1986, 1990, 1995), in particular, as presented by Bell (Bell 1987, p. 200). (See also the contribution of Ghirardi to this issue.)

18 Kochen 1985, Dieks 1991, and van Fraassen 1991.

19 Nelson 1966, 1985. (See also Goldstein 1987.)

20 Gell-Mann et al. 1993, Griffiths 1984, Omnes 1988. 


\section{References}

Albert, D.Z.: 1992, Quantum Mechanics and Experience, Harvard University Press, Cambridge, MA.

Bell, J. S.: 1964, 'On the Einstein-Podolski-Rosen paradox', Physics 1, 195-200. Reprinted in Bell 1987.

Bell, J. S.: 1966, 'On the problem of hidden variables in quantum mechanics', Review of Modern Physics 38, 447-452. Reprinted in Bell 1987.

Bell, J. S.: 1981, 'Quantum mechanics for cosmologists', in Quantum Gravity 2, C. Isham, R. Penrose, and D. Sciama (eds.), Oxford University Press, New York, pp. 611-637. Reprinted in Bell 1987.

Bell, J. S.: 1982, 'On the impossible pilot wave', Foundations of Physics 12, 989-999. Reprinted in Bell,1987.

Bell, J. S.: 1987, Speakable and unspeakable in quantum mechanics, Cambridge University Press, Cambridge.

Bell, J. S.: 1990, 'Against "measurement" ', Physics World 3, 33-40. [Also appears in 'Sixty-two Years of Uncertainty: Historical, Philosophical, and Physical Inquiries into the Foundations of Quantum Mechanics', Plenum Press, New York, pp. 17-31.

Beltrametti, E.G., Cassinelli, G.: 1981, The Logic of Quantum Mechanics, Reading, Mass..

Berndl, K., Dürr, D., Goldstein, S., Zanghì, N.: 1996, 'EPR-Bell Nonlocality, Lorentz Invariance, and Bohmian Quantum Theory', quant-ph/9510027, preprint. (To appear in Physical Review A, April 1996.)

Berndl, K., Dürr, D., Goldstein, S., Peruzzi G., Zanghì, N.: 1995, 'On the Global Existence of Bohmian Mechanics', Communications in Mathematical Physics 173, 647-673.

Birkhoff, G., von Neumann, J.: 1936, 'The logic of Quantum Mechanics', Ann. Math. 37.

Bohm, D.: 1952, 'A Suggested Interpretation of the Quantum Theory in Terms of 'Hidden' Variables, I and II,' Physical Review 85, 166-193. Reprinted in Wheeler and Zurek 1983, pp. 369-396.

Bohm, D., Hiley, B.J.:1993, The Undivided Universe: An Ontological Interpretation of Quantum Theory, Routledge \& Kegan Paul, London.

Daumer, M., Dürr, D., Goldstein, S. and Zanghì, N.: 1996, 'On the role of operators in quantum theory', in preparation.

Davies, E.B.: 1976, Quantum Theory of Open Systems, Academic Press, London.

De Witt, B. S., Graham, N. (eds.): 1973, The Many-Worlds interpretation of Quantum Mechanics, Princeton, N.J..

Dieks, D.: 1991, 'On some alleged difficulties in interpretation of quantum mechanics', Synthese 86, $77-86$.

Dürr, D., Goldstein, S. and Zanghì, N.: 1992, 'Quantum Equilibrium and the Origin of Absolute Uncertainty', Journal of Statistical Physics 67, 843-907; "Quantum Mechanics, Randomness, and Deterministic Reality," Physics Letters A 172, 6-12.

Dürr, D., Goldstein, S. and Zanghì, N.: 1996, 'Bohmian Mechanics as the Foundation of Quantum Mechanics', in Bohmian Mechanics and Quantum Theory: An Appraisal, J. Cushing, A. Fine and S. Goldstein (eds.), Kluwer Academic Press.

Everett, H.: 1957, 'Relative state formulation of quantum mechanics', Review of Modern Physics 29, 454-462. Reprinted in De Witt et al. 1973, and Wheeler et al. 1983. 
Gell-Mann, M. and Hartle, J.B.: 1993, 'Classical Equations for Quantum Systems', Physical Review D 47, 3345-3382.

Ghirardi, G.C., Rimini, A. and Weber, T.: 1986, 'Unified Dynamics for Microscopic and Macroscopic Systems', Physical Review D 34, 470-491.

Ghirardi, G.C., Pearle, P. and Rimini, A.: 1990, 'Markov Processes in Hilbert Space and Continuous Spontaneous Localization of Systems of Identical Particles', Physical Review A 42, 78-89.

Ghirardi, G.C., Grassi, R. and Benatti, F.:1995, 'Describing the Macroscopic World: Closing the Circle within the Dynamical Reduction Program', Foundations of Physics 23, 341-364.

Gleason, A. M.: 1957, 'Measures on the closed subspaces of a Hilbert space', Journal of Mathematics and Mechanics 6, 885-893.

Griffiths, R.B: 1984, 'Consistent Histories and the Interpretation of Quantum Mechanics' Journal of Statistical Physics 36, 219-272.

Goldstein, S.: 1987, 'Stochastic mechanics and quantum theory', Journal of Statistical Physics 47, 645667.

Jauch, J.M., Piron, C.: 1963, 'Can hidden variables be excluded in quantum mechanics?', Helvetica Phisica Acta 36, 827-837.

Jauch, J.M.: 1968: Foundations of Quantum Mechanics, Addison-Wesley, Reading, Mass..

Holland, P.R.: 1993, The Quantum Theory of Motion, Cambridge University Press, Cambridge.

Kochen, S., Specker, E. P.: 1967, 'The problem of hidden variables in quantum mechanics', Journal of Mathematics and Mechanics 17, 59-87.

Kochen, S.: 'A new interpretation of quantum mechanics', in Symposium on the Foundations of Modern Physics, P. Lathi and P. Mittelstaedt (eds.), World Scientific, Singapore.

Mott, N.F: 1929, Proceedings of the Royal Society A 124, 440.

Nelson, E.: 1966 'Derivation of the Schrödinger equation from Newtonian mechanics' Physical Review 150, 1079-1085.

Nelson, E.: 1985 Quantum Fluctuations, Princeton University Press, Princeton.

Omnès, R.: 1988, 'Logical Reformulation of Quantum Mechanics I', Journal of Statistical Physics 53, 893-932.

von Neumann, J.: 1932, Mathematische Grundlagen der Quantenmechanik Springer Verlag, Berlin. English translation: 1955, Princeton University Press, Princeton.

Schrödinger, E.: 1935, 'Die gegenwärtige Situation in der Quantenmechanik,' Die Naturwissenschaften 23, 807-812, 824-828, 844-849. [Also appears in translation as "The Present Situation in Quantum Mechanics," in Wheeler and Zurek 1983, pp. 152-167.]

van Fraassen, B.: 1991, Quantum Mechanics, an Empiricist View, Oxford University Press, Oxford.

Wheeler, J.A., Zurek, W.H. (eds.): 1983, Quantum Theory and Measurement, Princeton University Press, Princeton. 